\title{
UN GENIO DESFIGURADO: \\ CALDERÓN DE LA BARCA DURANTE EL FRANQUISMO
}

Laura Hernández González

Departamento de Literatura Española y Teoría de la Literatura

Universidad de Valladolid

Calle Plaza de Santa Cruz, 8

47002.Valladolid. España

laurah1987@hotmail.es

Nadie duda hoy día que Calderón de la Barca llegó a constituirse, durante todo el periodo franquista, como la encarnación literaria de los valores ideológicos del Régimen. De modo inusitado, Calderón y su obra adquirieron una nueva dimensión política y social; dejaron de ser historia, literatura o teatro para convertirse en símbolo, en emblema poético del conservadurismo, del patriotismo exaltado y del ultracatolicismo. Esta metamorfosis solo fue posible gracias a un poderoso aparato de propaganda que logró infiltrarse en todos los ámbitos sociales ligados a lo dramático: el educativo y académico, el editorial e, incluso, el propiamente teatral.

Ya a principios de siglo, en un ambiente de renovación dramática frente al anquilosado modelo realista, los pensadores de la derecha forjaron una nueva concepción de "Teatro Nacional», que el franquismo adaptará posteriormente. Entre estos teóricos destacan Ernesto Giménez Caballero y, sobre todo, Gonzalo Torrente Ballester. En efecto, «Razón y ser de la dramática futura» ${ }^{1}$, artículo del escritor gallego, sentará las bases

\footnotetext{
${ }^{1}$ Torrente Ballester, 1937.
} 
del futuro teatro franquista: un teatro que debía representar de modo conveniente y exaltado el catolicismo, el patriotismo y la gloria de la historia nacional.

En este contexto de innovación teatral, tanto los intelectuales de izquierdas como de derechas insisten en la necesidad de recuperación del patrimonio teatral del Siglo de Oro. Sin embargo, iniciada la Guerra Civil será el bando nacional el que con mayor frecuencia organice representaciones de teatro clásico en el frente entendiéndolo como síntesis dramática de lo español y lo católico. El teatro resulta un elemento propagandístico tan determinante durante el desarrollo de la Guerra Civil que, ya en noviembre de 1938, el bando nacional constituye la Junta Nacional de Teatros y Conciertos. Este organismo se transformaría, en 1940, en el Consejo Nacional de Teatros, encargado de administrar los Teatros Nacionales. También a finales de 1937 apareció el Teatro Nacional de la Falange, dirigido por Luis Escobar. En 1938, Dionisio Ridruejo se convierte en el director del Área de Prensa y Propaganda dentro de la España nacional y decide convertir el teatro en uno de los pilares fundamentales para la difusión de la ideología del nuevo régimen. Con este propósito promociona el auto sacramental ( $\mathrm{El}$ asunto de los Autos fue un disparate o capricho mío que no endoso a nadie» ${ }^{2}$ ), un subgénero didáctico que, a su juicio, sintetiza valores fundamentales para el franquismo como el catolicismo más ortodoxo y el patriotismo.

Acabada la contienda, el nuevo gobierno inició el proceso de creación de un llamado "Teatro Nacional» que sirviera a sus intereses ideológicos. Ello conllevó la apropiación por parte del franquismo del Teatro Clásico, asociado éste siempre a valores patrióticos, nacionalistas y ultracatólicos. Esta lectura «oficial» transformó las grandes obras de la dramaturgia áurea en un teatro de tesis, de ideas, elemental transmisor de la ideología establecida por las instancias gubernativas. Además, el régimen necesitaba crear sus propios mitos, grandes figuras históricas y artísticas universalmente reconocidas afines a su pensamiento. El héroe literario forjado fue, indudablemente, Calderón de la Barca. La elección no era casual, pues se sustentaba en todo el bagaje crítico reaccionario que había convertido en los siglos anteriores al dramaturgo en emblema del tradicionalismo y del catolicismo, dos ideales fundamentales para el franquismo. Concretamente, la autoridad incuestionable de Marcelino Menéndez Pelayo erigía a Calderón en encarnación plena de estos va-

${ }^{2}$ Ridruejo, 1976, p. 178. 
lores. Fernanda Andura Varela da cuenta de la tergiversación ideológica que padeció en estos años el teatro clásico y, en especial, la figura de Calderón de la Barca:

La imagen de los clásicos se vio afectada por una especie de burocratización, de exaltación oficial, traducida en un tratamiento escénico generalmente rutinario. El fondo ideológico de esta protección a los clásicos era bastante obvio. Significaba la defensa de unos valores culturales y espirituales ligados al nacionalismo español y a la memoria del Imperio.

Una imagen grandilocuente en cuanto al texto y católica en cuanto a la ideología prevalecía cada vez que se citaba el nombre de Calderón. Era, en términos escénicos, algo vinculado a los sermones, al teatro de tesis, a la espectacularidad gratuita, a la búsqueda de situaciones efectistas. El tratamiento atrevido de la escenografia, fundamental en la estética calderoniana, como desarrollo de la convención y el juego, fue utilizado en los primeros años de la postguerra con una desmesura y aparatosidad gratuitas ${ }^{3}$.

El teatro es, durante el franquismo, mucho más que un espectáculo: es un asunto de estado. En efecto, la dramaturgia es uno de los instrumentos propagandísticos más importantes del régimen y si bien el teatro puramente ideológico del bando nacional tendrá poca continuidad una vez acabada la Guerra Civil, el Teatro del Siglo de Oro se mantendrá a lo largo de toda la dictadura como una de las más eficaces herramientas de las instancias dominantes para propagar su concepción ideológica.

La universidad es uno de los ámbitos en los que el concepto de «Teatro Nacional» tuvo una mayor aceptación y difusión. Tras el exilio de un numeroso grupo de intelectuales de izquierda, el franquismo encontró resortes gubernamentales adecuados para orientar la investigación académica en la línea ideológica que trataba de establecer. El régimen difundió una imagen absolutamente idealizada del Siglo de Oro como periodo de esplendor católico e imperial. En este marco idílico había surgido la obra del mejor dramaturgo de nuestra literatura, Calderón de la Barca que habría logrado el máximo esplendor de la Comedia Nueva, al imbricarla de un espíritu plenamente español, tradicionalista y católico.

Las historias de la literatura publicadas en los años de la dictadura insisten en esta visión «oficial» de la dramaturgia calderoniana y, en ge-

\footnotetext{
${ }^{3}$ Andura Varela, 2000, p. 132.
} 
neral, de toda la literatura áurea. Resulta continua, asimismo, la referencia a Menéndez Pelayo como máxima autoridad en estas cuestiones, y ello hizo que su perspectiva reaccionaria y católica acerca de Calderón se difundiera durante buena parte del siglo $\mathrm{xx}$ en las Historias de la Literatura más autorizadas. Por ejemplo, la Historia de la Literatura de José Luis Alborg ${ }^{4}$ usa como fuentes para su capítulo sobre Calderón de la Barca fundamentalmente los estudios de Menéndez Pelayo y Valbuena Prat, lo que le lleva en una unívoca dirección crítica, ensalzando el profundo catolicismo del dramaturgo y considerando los autos sacramentales como las piezas más valiosas de su producción. Otis Green ${ }^{5}$, por su parte, considera al dramaturgo como la encarnación del catolicismo inherente al ser español. En el tomo dedicado a la Edad de Oro de Literatura de España de Francisco Ynduráin también aparece una visión tradicionalista del Siglo de Oro y de su literatura. En el prólogo al volumen dedicado a los siglos XVI y XVII Joaquín Entrambasaguas hace un breve resumen del mismo y presenta a Calderón como el mejor dramaturgo de la época (superior a Lope) y destaca los autos sacramentales como su mejor obra. Literatura de España constituyó un proyecto de historia literaria en el que se realizaba una antología de textos significativos de la literatura española con sus correspondientes estudios. Resulta muy curioso observar que los textos calderonianos seleccionados son La dama duende y un auto sacramental, El veneno y la triaca. Si bien el primero continúa siendo una obra bastante apreciada, la aparición de $E l$ veneno y la triaca demuestra la alta valoración que durante la dictadura recibieron los autos sacramentales. En el comentario que precede al texto del auto, Eugenio Frutos afirma que «no hace falta mencionar su catolicidad y su españolismo, a pesar del carácter universal $»^{6}$. De nuevo, los intelectuales de la época franquista insisten en la ligazón entre la obra calderoniana, el catolicismo y un sentido muy determinado de la «españolidad", entendida en términos tradicionales y reaccionarios.

Desde la universidad, esta visión de la obra de Calderón se transmitió a la enseñanza media. Tomando como muestra algunos libros de texto del periodo franquista, se comprueba que trasmiten información un tanto sesgada de Calderón de la Barca, tanto de sus circunstancias biográficas como de su producción dramática con el objetivo de construir

\footnotetext{
${ }^{4}$ Alborg, 1967.

${ }^{5}$ Green, 1969.

${ }^{6}$ Frutos, 1972, p. 569.
} 
una imagen ultracatólica y profundamente patriótica del dramaturgo. Así, por ejemplo, se insiste en la educación jesuítica y en la gran formación teológica de Calderón, así como en el hecho de que se ordenara sacerdote o en su experiencia militar. Se omite, sin embargo, el hecho de que estuvo casado y tuvo descendencia. En lo relativo a su obra se destaca a Calderón, ante todo, como el gran genio del auto sacramental español y su gran obra, La vida es sueño, se interpreta únicamente en clave cristiana. Apenas se mencionan sus comedias de tono festivo o sus aportaciones al teatro breve barroco, otorgando a la obra calderoniana un carácter profundamente serio y moralizante, en términos católicos, y de defensa de la virtud y el honor.

Asimismo, Historia de la Literatura. Sexto curso de la editorial Teide establece una comparación un tanto simplista con Lope de Vega, cuyo teatro se presenta como mucho más «fácil» e «intrascendente».

A través de todos estos recursos, la escuela franquista transmitió al conjunto de la sociedad una imagen manipulada de la obra calderoniana, primando sus aspectos católicos, teológicos y patrióticos y asociándolos indisolublemente con la ideología dominante durante la dictadura.

Por otra parte, uno de los criterios que nos permite establecer la canonicidad de una obra literaria es su presencia en las colecciones de clásicos. Así, el lector de un clásico espera encontrar una obra socialmente reconocida y valorada por su calidad estética, artística e ideológica. Las colecciones están indudablemente ligadas al campo universitario, su generador y principal receptor, pero también lo trascienden, llegando a la ciudadanía en general. Por ello, la consideración de una obra literaria como clásico es el resultado de un complejo proceso en el que el ámbito académico y el editorial se encuentran en constante retroalimentación. Sin embargo, las colecciones de clásicos no están exentas de las exigencias del mercado literario, lo que hace que los títulos más populares sean continuamente reeditados, afianzándose en el canon social y otros, en cambio, terminen por desaparecer. Por ello, la configuración de las colecciones de clásicos resulta históricamente variable. De este modo, revisando los índices de las principales colecciones de clásicos que circulaban en España durante el franquismo, podemos determinar qué noción poseía la sociedad de Calderón: cuáles de sus obras se consideraban canónicas y qué textos se leían.

La primera colección de clásicos que analizaremos será la «Biblioteca de Autores Españoles» (BAE). Se trata de un proyecto que, pese a ini- 
ciarse en el siglo xIX, en 1846, mantuvo durante el periodo franquista su presencia en las bibliotecas españolas pues había constituido el primer intento sistemático de ofrecer ediciones cuidadas de los clásicos españoles. La BAE reedita para sus lectores un total de cuatro tomos en los que recoge las ediciones de Hartzenbusch de comedias calderonianas y, además, un monográfico de González Pedroso, Autos sacramentales desde su origen hasta fines del siglo XVII, en el que también encontramos textos del dramaturgo. Esta colección mantiene así, entre los lectores del franquismo, una visión decimonónica de la figura de Calderón, que valora especialmente sus autos. Asimismo, se dedica el mismo espacio en la colección a su obra que a la de Lope de Vega pese a que la de este último es cuantitativamente mucho mayor. De algún modo, la dinámica de publicación de la BAE nos hace pensar que a finales del siglo XIX y principios del xx, quizá por la influencia del calderonismo alemán, la figura de Calderón de la Barca era más apreciada que la de Lope. Esta sensación de primacía de Calderón se trasmitirá a los lectores que manejen los textos de la BAE durante el franquismo, así como la importancia destacada que en la colección reciben los autos sacramentales frente a otros subgéneros dramáticos. Igualmente importante para el desarrollo de la Filología en España resulta el nacimiento de la colección «Clásicos Castellanos», un proyecto engendrado en 1910 en el Centro de Estudios Históricos, bajo la dirección de Ramón Menéndez Pidal. La presencia de obras de Calderón de la Barca en la colección de «Clásicos Castellanos» es muy abundante, lo que da una idea exacta de la gran valoración que recibió el dramaturgo a principios del siglo $\mathrm{xx}$, en una colección que también sería muy leída durante la dictadura. En este periodo la colección pasaría a ser propiedad de la editorial Espasa Calpe, que continuaría el proyecto de publicación de textos clásicos hispánicos.

A través de la consulta bibliográfica de catálogos de diversas bibliotecas que conservan la colección "Clásicos Castellanos» podemos hacernos una idea de cuáles fueron los textos de Calderón más aclamados y editados durante el franquismo. Y en efecto, estos son sus autos sacramentales, publicados en 1926 por Ángel Valbuena Prat y reeditados hasta en seis ocasiones en el transcurso del régimen. El volumen 123, que incluye las comedias religiosas de Calderón La devoción de la Cruz y El mágico prodigioso, también gozó de gran aceptación entre los lectores del periodo franquista, pues encontramos tres reediciones del mismo. El honor calderoniano, asociado a la virtud cristiana y al patriotismo, 
fue un aspecto muy valorado durante el franquismo y, por ello, no es de extrañar que "Clásicos Castellanos» creara un compendio de comedias calderonianas de capa y espada y otro de dramas de honor.

Paradójicamente, algunos de los hoy considerados textos indiscutiblemente canónicos del teatro calderoniano, como La vida es sueño o El alcalde de Zalamea, no contaron con una primera edición en la colección hasta 1955, es decir, casi treinta años después de la publicación del volumen destinado a los autos sacramentales. El tomo conjunto que contenía La vida es sueño y El alcalde de Zalamea fue, no obstante, reeditado en 1960, 1964, 1968 y 1971, es decir, en el momento en el que el régimen cedió un poco en su voluntad de exaltación patriótica. Por otro lado, una obra hoy tan valorada como El príncipe constante no aparece en la colección hasta 1975.

El análisis de la colección "Clásicos Castellanos», la más autorizada en la época desde el punto de vista filológico, denota que, en el ámbito académico del primer franquismo, se prestó especial atención al teatro calderoniano genuinamente religioso y, en particular, sus autos sacramentales.

Otra de las colecciones de clásicos más importantes en la época fue "Austral», la primera en formato de bolsillo que, con un precio muy reducido, difundió las grandes obras de nuestra literatura entre los sectores más populares. En ella se incluyeron, por supuesto, algunas de las grandes obras de Calderón de la Barca pero la selección difiere de la nómina que componía «Clásicos Castellanos». Ello puede deberse al carácter más popular de una colección, alejada del ámbito académico que, para satisfacer a su público, incluía obras calderonianas de carácter más lúdico. De este modo, encontramos cuatro ediciones de un volumen que contenía una comedia de enredo y otra de santos, Casa con dos puertas mala es de guardar y El mágico prodigioso entre 1942 y 1961. El gusto popular por la vertiente más festiva de la producción calderoniana se observa asimismo en las cuatro ediciones de las que fue objeto un tomo que contenía $A$ secreto agravio, secreta venganza y La dama duende y en la publicación en 1970 de la edición conjunta de Guárdate del agua mansa y Amar después de la muerte.

Asimismo, los autos sacramentales calderonianos aparecieron publicados en esta colección de bolsillo en cinco tiradas durante el régimen franquista, lo que demuestra que este tipo de obras se intentaba difundir entre un público menos especializado. Los dramas de honor estaban 
representados en la colección por un volumen que contenía No hay burlas con el amor y El médico de su honra y que fue reeditado en cuatro ocasiones. A diferencia de lo que ocurría en «Clásicos Castellanos», $E l$ príncipe constante (publicado junto a El mayor monstruo del mundo) contó con una edición temprana en 1945 que volvió a reaparecer en dos ocasiones durante la dictadura.

Pero, sin lugar a dudas, el gran éxito calderoniano de la colección "Austral» fue el volumen en el que se incluían dos de sus obras más universales: El alcalde de Zalamea y La vida es sueño. Dicho tomo contó con un total de dieciocho tiradas en los años del franquismo, lo que da idea de su increíble popularidad. Algo similar ocurre en otra de las colecciones de bolsillo más populares de mediados del siglo xx, la editada conjuntamente por Salvat y RTVE. En efecto, entre los títulos de esta colección, de clara orientación popular y enorme éxito entre la juventud de la época, encontramos únicamente un volumen con obras calderonianas que contiene precisamente La vida es sueño y El alcalde de Zalamea, editadas por Francisco Ruiz Ramón. Parece ser que el libro resultó del gusto del público, como demuestra el hecho de que se realizaran cuatro tiradas en tan solo un año, entre 1970 y 1971.

Un análisis concienzudo de las obras calderonianas publicadas en las colecciones de clásicos de carácter popular durante el periodo franquista revela ciertas diferencias en las preferencias lectoras del público académico, más inclinado al análisis e interpretación de los textos teológicos, en la línea de la orientación ideológica dominante, frente a las del público popular, que gustaba más de obras de tono más distendido y festivo.

El teatro clásico en general y el calderoniano en particular gozó de una presencia ininterrumpida en los escenarios durante la dictadura. A partir del concepto de Teatro Nacional acuñado por los ideólogos franquistas y como consecuencia de la identificación de esta concepción con el Teatro del Siglo de Oro, este resurgió con vigor en los teatros públicos y privados durante el régimen.

Durante la Guerra Civil, el teatro clásico se representa abundantemente en los «teatros de urgencia» tanto del bando nacional como del republicano, teatrillos improvisados en el frente o en las ciudades en los que se ponen en escena obras con un propósito ideológico claro, según los ideales políticos de cada bando. 
Curiosamente, en este ambiente bélico, Calderón será uno de los autores más representados, sobre todo por los actores del bando nacional, que lo erigen como emblema del catolicismo, el patriotismo español y la gloria imperial. De este modo, el Teatro Nacional de la Falange Tradicionalista y de las JONS, creado en 1937 y dirigido por Luis Escobar, realizará una gira por todas las capitales de la zona nacional representando obras clásicas, entre las que podemos destacar La vida es sueño.

Tras el fin de la guerra, la cultura oficial impone un repertorio concreto sometido a una lectura ideológicamente interesada y desvirtúa el sentido de la producción dramática calderoniana, al aplicarle criterios de pensamiento que se relacionan con la realidad social y política de la España franquista pero no con la del siglo XVII, la de la época en la que estos textos fueron escritos. Si bien toda representación de un texto clásico implica, en cierta medida, su relectura y actualización, el franquismo pervirtió este proceso al focalizar la atención de espectadores y críticos exclusivamente en los aspectos de la obra calderoniana que respondían a la imagen artificial e icónica que habían creado del dramaturgo y dejando de lado o rechazando todos aquellos que contravinieran este constructo oficialista.

Las obras del corpus calderoniano que mejor responden a las necesidades propagandísticas del gobierno franquista son, primeramente, los autos sacramentales, por su exaltación del catolicismo y su carácter didáctico y, seguidamente, La vida es sueño y El alcalde de Zalamea, sometidas, eso sí, a una interpretación exclusivamente en clave cristiana que deja de lado sus aspectos filosóficos, políticos o sociales. Así las cosas, aunque en esta época encontremos cierto auge de las obras calderonianas sobre los escenarios, no puede decirse que socialmente o desde el ámbito especializado se avance demasiado en el conocimiento de la obra de Calderón pues lo que el régimen propugna es una lectura sesgada y manipulada de la misma.

Desde sus inicios, Calderón de la Barca fue el dramaturgo favorito del régimen y sus piezas enmarcaron los actos oficiales más trascendentales de la España franquista. Ya en la celebración de la Victoria, el Teatro Nacional de la FET y las JONS representó precisamente un auto sacramental de Calderón, La cena del rey Baltasar, en el Retiro. Un año después, en el primer aniversario de laVictoria, la Delegación Nacional de Prensa y Propaganda organizó una auténtica fiesta teatral barroca en 
el Teatro Español, a imitación de las celebradas en nuestro Siglo de Oro. El espectáculo titulado España, una, grande y libre poseía la estructura de una representación del siglo xviI, pues contó con la Loa famosa de la unidad de España, la Comedia heroica por la libertad de España y una farsa titulada Fiesta alegórica de la grandeza de España. Entre otros elementos barroquizantes, la puesta en escena contó con una pieza musical, El jardín de Falerina, compuesta en 1629 por Joseph Peiró precisamente como acompañamiento a una loa de Calderón y de escenas alegóricas y artificios similares a los de la dramaturgia áurea.

La creación de los teatros nacionales, el Español y el María Guerrero, responde igualmente al deseo del gobierno de Franco de constituir un nuevo modo de hacer teatro afin a sus ideales políticos, religiosos y sociales. El Teatro Español, encargado del repertorio dramático clásico, será el instrumento transmisor de esta cultura «oficial», de este modo particular y políticamente interesado de leer a los escritores áureos y especialmente a Calderón de la Barca. Por ello, no es de extrañar que durante la dictadura sus obras proliferen en la cartelera del Español.

Cayetano Luca de Tena, que reemplazó a Felipe Lluch al frente de este espacio escénico, programó ya en 1942 La dama duende, uno de los textos más atractivos de Calderón. Tan solo un mes después, se estrenó el montaje de El pleito matrimonial del Alma y del Cuerpo en los Jardines del Retiro. El interés del director por el subgénero sacramental pone de manifiesto la orientación ideológica que las representaciones de teatro clásico estaban tomando en España. El exitoso espectáculo, con trabajo escenográfico de Sigfrido Burmann se repuso en la celebración del Día de la Hispanidad y de nuevo en 1943, esta vez con una pieza breve calderoniana añadida, La mojiganga de la muerte. El Teatro María Guerrero, que más adelante se especializaría en un repertorio de teatro contemporáneo, comenzó sin embargo su andadura en 1940 con una representación calderoniana: un espectáculo compuesto por el entremés La rabia y el auto sacramental La cena del rey Baltasar bajo la dirección de Claudio de la Torre y Luis Escobar. Este modo de inaugurar uno de los espacios escénicos más importantes durante la dictadura no deja lugar a dudas de la trascendencia ideológica que el régimen otorgaba a los autos sacramentales de Calderón. Quizá por ello La cena del rey Baltasar fue una de las obras seleccionadas para representar a la «nueva» España de Franco en la Exposición del Mundo Portugués. 
En los años 40 el María Guerrero iniciaría la tradición de representar La vida es sueño en la temporada otoñal. También continúan su andadura los Grupos de Teatro Universitario (TEU) que habían comenzado su actividad durante la II República. Modesto Higueras, al frente de uno de ellos, pone en escena La vida es sueño, en 1944. Un año después llevaría a las tablas del María Guerrero una pieza poco conocida del repertorio calderoniano: El santo rey don Fernando, obra que alude a una etapa gloriosa en la defensa del catolicismo en España, la Reconquista. Además, en 1948, la Asociación de Prensa patrocinó un espectáculo popular en el Teatro del Progreso que incluía, entre otras obras, El alcalde de Zalamea, dirigida por Luis Escobar.

Por su parte el Teatro Español montó dos piezas más de Calderón a finales de los 40 y principios de los 50, bajo la dirección de Luca de Tena: El médico de su honra y El alcalde de Zalamea. El cese del director al frente del Español sume a los teatros oficiales en un periodo de crisis, tras la que se encarga a José Tamayo la dirección del Español y a Claudio de la Torre, la del María Guerrero. La llegada de Tamayo al Español supone una auténtica modernización en la manera de concebir y representar el teatro clásico hispánico y, especialmente, las piezas de Calderón de la Barca. No en vano, José Tamayo había volcado su carrera como director, productor y empresario teatral en la representación de obras clásicas al frente de la Compañía Lope de Vega. De hecho, uno de sus espectáculos más memorables fue la representación del auto sacramental El gran teatro del mundo, en el Teatro de la Comedia y al aire libre, frente al Palacio Real, en la festividad de San Isidro de 1952.

Este éxito en el montaje de una obra tan compleja, en lo formal y en lo ideológico, como un auto sacramental hizo que la compañía Lope de Vega fuera la seleccionada por las autoridades para el montaje de otro auto calderoniano, La cena del rey Baltasar, que había de ser representado como homenaje de España al papa Pío XII en el Vaticano. La temática y la orientación religiosa de los espectáculos de Tamayo les valieron el reconocimiento de las autoridades franquistas, que no dudaron en reconocer su labor, colocándolo al frente del Español. Siguiendo con su afición por la producción calderoniana, el director realizó una reposición, en 1954, de su éxito La cena del rey Baltasar y una versión de La hidalga del valle. En 1955, dirigió un nuevo montaje de La vida es sueño en el que participaron actores de la talla de Francisco Rabal, Mary Carrillo o Asunción Balaguer. Esta versión de La vida es sueño, realizada 
por Nicolás González Ruiz giró dentro del repertorio del proyecto Festivales de España e, incluso, llegó a representarse en el Teatro de las Naciones de París.

También en 1955 José Tamayo retoma el subgénero sacramental con una versión de El pleito matrimonial del alma y el cuerpo realizada por Nicolás González Ruiz, uno de sus adaptadores predilectos. Habrá que esperar hasta 1959 para que los versos calderonianos resuenen sobre las tablas del Teatro Español, en una refundición de José María Pemán de Los encantos de la culpa, interpretada, entre otros, por Irene López de Heredia o Asunción Sancho. José Tamayo participó activamente en el proyecto Festivales de España, que trataba de difundir la cultura oficial por todos los rincones de nuestro país y que, como mecanismo educativo y propagandístico del régimen franquista abundó en la representación de obras de Calderón, insistiendo en aquellas que mejor ilustraban los valores de la cultura oficial: catolicismo, patriotismo, nacionalismo... De este modo, el director giró su espectáculo El gran teatro del mundo por la Plaza Porticada de Santander, la Plaza de las Pasiegas de Granada y el Teatro Romano de Mérida. En 1962,Tamayo abandonó la dirección del Teatro Español pero no por ello decayó su interés por la representación de piezas calderonianas ya que en 1963 revisó el mayor éxito de su carrera escénica, La cena del rey Baltasar. El montaje se incorporó también al proyecto Festivales de España y se representó en diecisiete localidades españolas, alcanzando su mayor grado de espectacularidad en el Patio del Monasterio de El Escorial.

Además de los grandiosos montajes de Tamayo, Festivales de España hizo girar por la Península otras muchas propuestas espectaculares construidas a partir de textos calderonianos. Es el caso de los montajes de Cayetano Luca de Tena Mañanas de abril y mayo y No hay burlas con el amor, que supusieron una incursión por parte del director en territorios dramáticamente inexplorados dentro de la producción calderoniana. Algo similar puede señalarse a propósito de El jardín de Falerina, dirigido por Juan Germán Schroeder. Otros directores, en cambio, giraron montajes de títulos consagrados como La dama duende de Modesto Higueras o La devoción de la cruz y La hidalga del valle de Gustavo Pérez Puig.

Los años 60 suponen un punto de inflexión en la vida cultural y escénica española. Las tensiones políticas y sociales en las que se halla sumido el país así como el surgimiento de numerosos núcleos de oposición al régimen franquista causan importantes cambios en el ámbi- 
to teatral. Tanto el público como las compañías buscan nuevos títulos en las carteleras y el movimiento teatral universitario, el TEU, se erige como un espacio de oposición política, social y artística a la dictadura de Franco. Las obras de Calderón continúan poniéndose en escena en los teatros españoles, pero su presencia es cada vez más espaciada e irregular puesto que la manipulación ideológica a la que fueron sometidas en los primeros años del régimen hace que el público de los 60 las rechace por sus implicaciones políticas.

Este rechazo a la visión franquista de Calderón se manifiesta en 1963, cuando Cayetano Luca de Tena inicia la temporada en el Teatro Español con No hay burlas con el amor, una comedia que rompe radicalmente con la imagen de autor serio, profundo y ultracatólico que la dictadura había presentado del autor. La novedad en el planteamiento del texto hizo que la obra obtuviera un gran éxito de taquilla, lo que llevó el montaje al Teatro Griego de Montjuich y a otras diecisiete localidades españolas, en el marco del proyecto Festivales de España. Paralelamente, la compañía encabezada por la actriz Mary Carrillo giró por diversas capitales españolas con un espectáculo basado en Dar tiempo al tiempo, alejándose de nuevo del repertorio calderoniano que el régimen había establecido como canónico.

En el año 1965 este propósito de ruptura con la imagen reaccionaria tradicionalmente asociada a la figura de Calderón llevó a Humberto Pérez de la Ossa a embarcarse en un arriesgado proyecto en el que se entremezclaba El alcalde de Zalamea con un sainete de Ramón de la Cruz, El Manolo.

Ya en 1966, Calderón renace en el Teatro Español de la mano de José Luis Alonso Mañes, quien dirigió La dama duende y realizó una gira veraniega dentro del proyecto Festivales de España. El espectáculo consiguió buenas críticas pero en ellas se observa un llamativo cambio de actitud: se insiste en la necesaria renovación de los montajes de teatro clásico para que este deje de concebirse como objeto arqueológico portador de verdades y valores eternos e incuestionables y comience a ser entendido como un objeto artístico capaz de seguir conectando con el público de la época, en tanto en cuanto, presenta un conflicto, político, social, religioso o sentimental que continúa siendo motivo de cuestionamiento y reflexión personal para el espectador contemporáneo.

A finales de los años 60, el teatro, tal y como había sido concebido en los años de la dictadura franquista, entra en declive, pues la vida escénica 
no puede permanecer ajena a la crisis política, social e ideológica que azota el país. La nueva Ley de Prensa de 1966, que suprime la censura, hace que el interés de los hombres de teatro se vuelva hacia aquellos dramaturgos y obras que hasta entonces habían sido vedados por el sistema. El teatro áureo, y especialmente la figura de Calderón, aparecen ligados a unos valores reaccionarios que no se corresponden con el cambio que el espectador español anhela en su teatro y en su sociedad. Desde finales de los 60 y durante todo el proceso político de la transición a la democracia, Calderón se verá desplazado de los escenarios. Asimismo, el incendio del Teatro Español de Madrid, el 19 de septiembre de 1975, y la demora de más de cuatro años en su rehabilitación no contribuyeron al resurgimiento del teatro clásico, que experimentaba una progresiva decadencia, tanto en el ámbito de la Filología como en el del espectáculo. Tampoco el hecho de que el proyecto Festivales de España terminara por ser desmantelado, debido al derrocamiento de los ideales que durante años habían sustentado estas giras veraniegas. En el ámbito académico, la crítica se distancia del estudio de una figura, la de Calderón, que se siente reaccionaria y antipática, indisolublemente ligada al pensamiento franquista. Aunque esta circunstancia ha cambiado en los últimos años, sobre todo a raíz del aniversario de la muerte del dramaturgo, en 1988, fecha en la que comenzó a revalorizarse su figura a través de una oleada de representaciones y estudios de sus obras, parte de la sociedad española quizás sigue relacionando el teatro de Calderón con una perspectiva reaccionaria de la realidad, asociada al patriotismo y el catolicismo exaltados propios de la ideología del primer franquismo.

A través de este estudio hemos tratado de analizar las claves del proceso a través del cual el franquismo ideologizó la figura de Calderón de la Barca para convertirla en emblema del régimen y de los valores católicos y tradicionalistas que este defendía. Esta imagen artificial de Calderón como adalid del catolicismo y del tradicionalismo más reaccionario ha influido de modo determinante en el estudio e interpretación de su obra. Se hace, por tanto, necesario estudiar los mecanismos que el franquismo empleó para crear en el imaginario colectivo español una asociación indisoluble entre la obra calderoniana y los valores del régimen y demostrar que esta visión de los textos del dramaturgo áureo es, en realidad, una construcción ideológica ajena a los contenidos intrínsecos de la misma. Mediante este análisis hemos pretendido dilucidar qué ideas y valores tradicionalmente asociados a la producción 
calderoniana son, en realidad, añadidos ideológicos, acordes con una situación histórica, política y social tan peculiar en nuestra historia como fue la del régimen franquista. Solo así podremos penetrar, sin prejuicio heredado alguno, en las profundidades artísticas y dramáticas de uno de los grandes legados de la literatura universal: el teatro de Calderón de la Barca. 


\section{Bibliografía}

Alborg, José Luis, Historia de la Literatura Española, vol. II. Época Barroca, Madrid, Gredos, 1967.

Álvarez Barrientos, Joaquín, «Pedro Calderón de la Barca en los siglos XviII y xIX. Fragmentos para la historia de una apropiación», en Estado actual de los estudios calderonianos, ed. Luciano García Lorenzo, Kassel, Reichenberger, 2000, pp. 279-324.

Andura Varela, Fernanda, "Calderón en la escena española, 1900-2000», en José María Díez Borque y Andrés Peláez Martín, Calderón en escena: siglo XX, Madrid, Comunidad de Madrid, 2000, pp. 123-156.

Checa, Julio, «El teatro español durante los años 50», en Historia de los Teatros Nacionales 1929-1962, Madrid, Ministerio de Cultura, 1993, pp. 106-119.

Entrambasaguas, Joaquín de, «Prefacio», en Domingo Ynduráin, Literatura de España. Edad de Oro, tomo II, Madrid, Editora Nacional, 1972, pp. 9-17.

Frutos, Eugenio, «La dama duende. El veneno y la triaca», en Domingo Ynduráin, Literatura de España. Edad de Oro, tomo II, Madrid, Editora Nacional, 1972, pp. 564-574.

Green, Otis, España y la tradición occidental. El espíritu castellano desde el «Cid» hasta Calderón, Madrid, Gredos, 1969.

Iglesias Feijoo, Luis, "Calderón ayer y hoy. Sobre el origen romántico de la visión actual de Calderón», en Proyección y significados del Teatro Clásico Español. Congreso Internacional: homenaje a Alfredo Hermenegildo y Francisco Ruiz Ramón. Madrid, ed. José María Díez-Borque y José Alcalá-Zamora, Madrid, Sociedad Estatal para la Acción Cultural Exterior, 2004, pp. 150-172.

Meregalli, Franco, «Consideraciones sobre tres siglos de recepción del teatro calderoniano", en Calderón. Actas del Congreso Internacional sobre Calderón y el Teatro del Siglo de Oro, ed. Luciano García Lorenzo, Madrid, Consejo Superior de Investigaciones Científicas, 1983, vol. I, pp. 103-124.

Peréz Magallón, Jesús, Calderón: icono cultural e identitario del conservadurismo político, Madrid, Cátedra, 2010.

Ridruejo, Dionisio, Casi unas memorias, Barcelona, Planeta, 1976.

Torrente Ballester, Gonzalo, «Razón y ser de la dramática futura», Jerarquía, 2, 1937, pp. 61-80. 